\title{
Description of Epochs of 02 Desaturation Less than 92\% Greater than 5 Minutes Prior to Organ Excision
}

National Cancer Institute

\section{Source}

National Cancer Institute. Description of Epochs of O2 Desaturation Less than 92\%

Greater than 5 Minutes Prior to Organ Excision. NCI Thesaurus. Code C159272.

A textual representation of the information regarding an $\mathrm{O} 2$ desaturation epoch of less than 92 percent, lasting greater than 5 minutes, prior to organ excision. 